\title{
Assessing Spatial Configurations and Transport Energy Usage for Planning Sustainable Communities
}

\author{
Remco de Koning ${ }^{1}\left(\mathbb{D}\right.$, Wendy Guan Zhen Tan ${ }^{1,2} \mathbb{D}$ and Akkelies van Nes ${ }^{1,3, * \mathbb{C}}$ \\ 1 Department of Civil Engineering, Western Norway University of Applied Sciences, 5020 Bergen, Norway; \\ REK@hvl.no (R.d.K.); WTA@hvl.no (W.G.Z.T.) \\ 2 Landscape Architecture and Spatial Planning, Wageningen University and Research, \\ 6708 PB Wageningen, The Netherlands \\ 3 Department of Urbanism, Faculty of Architecture, TU-Delft, 2628 BL Delft, The Netherlands \\ * Correspondence: a.vannes@tudelft.nl
}

Received: 12 September 2020; Accepted: 29 September 2020; Published: 2 October 2020

check for updates

\begin{abstract}
Energy usage in cities is intertwined with its spatial configuration-the denser and more compact the city, the more concentrated and efficient the energy usage is to be expected. To achieve sustainable communities, cities (and their inhabitants) must reconsider its spatial configurations in the context of rapid urbanisation and growth in light of limited resources and conflicting spatial claims. This article seeks to understand how spatial configurations affect transport energy usage in cities and propose an integrated assessment approach factoring spatial configurational analysis in relation to transport energy usage at the micro- and macroscale. Comparing Bergen, Norway, and Zürich, Switzerland, findings showed that spatial configurations were positively correlated to transport energy usage. Street structures suitable for walking and less suitable for car traffic tended to exhibit lower amounts of energy usage. Following this, nine typologies of transport and land use patterns are described to support planning for more sustainable means of transport.
\end{abstract}

Keywords: transport energy usage; sustainable mobility; space syntax; natural movement; natural urban transformation

\section{Introduction}

Challenges to sustainable development in the form of rapid urbanisation and the emergence of very dense megacities and metropolises have increased pressure on land use and transportation networks and corresponding energy resource limitations [1]. As cities grow, the need to maintain a feasible level of service in the transportation network while ensuring liveability increases with mounting difficulties. Urban planners and city managers have to balance the need for efficient traffic flow and increased network capacity as urban areas increase in size while ensuring that they still function at a human scale to offer accessibility to all.

In addition to the challenges of complexity and competition for space, the global threat of climate change has led to an escalating urgency to reduce our ecological footprint and make responsible choices regarding our decisions and the goods and services we use. The transport sector is responsible for $20 \%$ of EU-28 greenhouse gas emissions (excluding international aviation and maritime emissions) [2] and is estimated to account for anywhere from between $20-50 \%$ to one-third of its total energy usage depending on the source. It is important to reconsider how we assess and structure our cities to make them less car-oriented and translate this into actionable knowledge for sustainable development.

The recent Sustainable Development Goals (SDGs) are indicative of the challenge above [3]. Although set-up as stand-alone goals, each SDG has an explicit and implicit relationship with each other. Take for example SDG 11-Make cities and human settlements inclusive, safe, resilient, 
and sustainable-where the focus is on building cities and communities that are equitable but also sustainable, and SDG 7-Ensure access to affordable, reliable, sustainable, and modern energy for all-where the focus is on energy resource resilience and robustness. This is where a fine-tuning of the current assessment methodologies is needed if cities are expected to embrace comprehensive strategies to reduce fluctuations in energy resources or decreased levels of services.

Concerning the relationship between urban form and energy use, there has been a multitude of research confirming the classic Newman and Kenworthy [4] study on how high-density environments have lower energy usage [5-7]. However, there is a need to understand how different spatial configurations affect transport energy usage and how that can be assessed beyond limited focus on either building units or at a regional scale [5]. This knowledge is needed for planning transport infrastructures that can facilitate and encourage sustainable mobility and reduce energy consumption. This assessment requires a combination of expertise and methods typically separated by domains of traffic engineering which calculate traffic flow and energy use for transport, architects, and urban designers, who analyse urban space and form and spatial planners who can translate indicators into feasible policy.

This article combines the above disciplines to ask the following research questions:

1. How can urban form and movement theories contribute to an integrated assessment methodology for sustainable communities and inform urban planning policy and practice?

2. Which spatial configurations affect transport energy usage of private vehicles in cities and what are the categories observed?

Using the cases of Bergen, Norway, and Zurich, Switzerland, this article presents an analysis of urban spatial configuration (street structures) based on the theory of the natural movement economic process $[8,9]$ with space syntax analyses in relation to transport energy usage modelling with MATSim. These variables are spatially correlated in GIS (Geographical Information Systems) to inform how the spatial configuration of the built environment can influence transport energy usage at the local and the regional scale. We propose to assess six variables (see Table 1) at the micro- and macroscales that inform spatial configurations.

Our findings showed that the relationship between spatial configuration and transport energy usage depends on the various degrees of inter-accessibility at various scale levels, from the local (micro) scale to the city-wide (macro) scale. Comparing both cities, positive and significant co-relations were observed between streets with the space syntax variable "angular choice" with a high metrical radius (macro scale. i.e., suitable for car traffic) and transport energy usage. Our analysis identified nine typologies of spatial configurations through aggregating route choice variables and indicates their suitability in reducing transport energy consumption. This article concludes by proposing transport and land use planning strategies based on these nine typologies to reduce transport energy usage and achieve sustainable urban forms.

\section{Theoretical Framework}

Urban form has a direct impact on building and transport energy usage and these factors are directly affected by urban planning policies [10] (p. 3). This mutual relationship has been widely researched since the late 1980s [5,6,10-12]. The implementation gap lies in the coupling of an understanding of this relationship at both the local (micro) scale and regional scale [11] (see Figure 1). Here, we turn to Rådberg [13] who studied how a fine-grained deformed street network contributes to lower energy usage for transport and described the density paradox wherein two concepts for the ideal sustainable city clash-the compact city versus the green city. The former implies the sharing of infrastructure, space, and facilities, thereby reducing the total footprint per capita, and the latter has connotations of attractiveness and well-being through its "green" spaces for cultivation, water infiltration, and recreation. The normative nature of these sustainable concepts leaves us lacking the descriptive precision needed to assess and implement an ideal sustainable urban form [13] (p. 385). 
Jabareen's [12] review of what these forms could be, identified four types: compact city, eco-city, neotraditional development, and urban containment. However, within these types, the issue of sustainable transport still remained one of the largest challenges $[6,10]$.

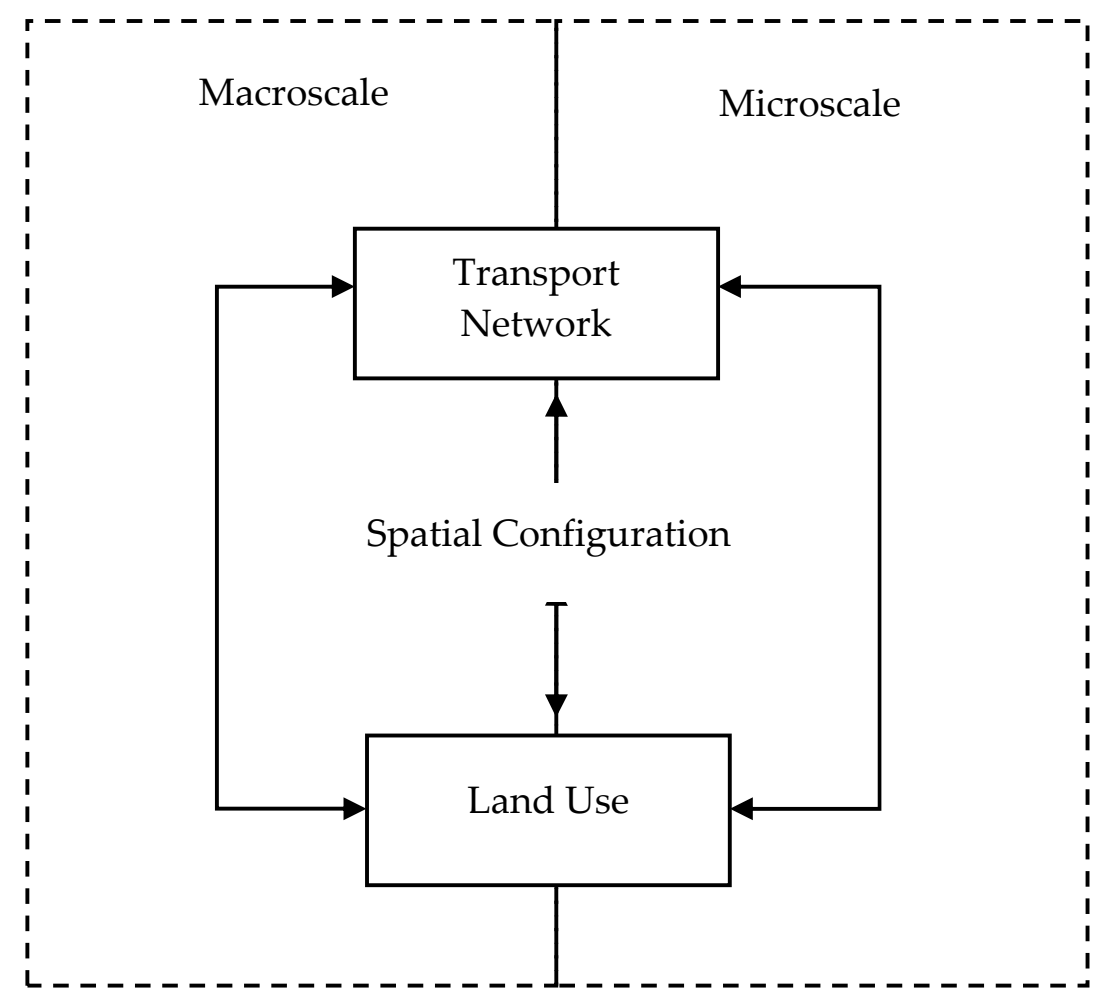

Figure 1. Conceptual framework for understanding spatial configuration.

\subsection{Transport and Land Use Integration at the Macro- and Microscales}

If achieving sustainable cities and communities is the goal, then there is an urgency to adopt integrated policies and plans towards inclusion and resource efficiency (SDG 11.B). There is a need to understand how transport and land use integration can play a role. To do so, access to safe, affordable, accessible, and sustainable transport systems for all must be provided (SDG 11.2). The necessary infrastructure will need to be upgraded to make them sustainable (SDG 9.4) [3]. This holistic approach was also advised as a key ingredient of achieving sustainable mobility [14,15]. A chief culprit to unsustainable transport impact is the use of the private vehicles. Hence, this article will focus on the energy usage related to car usage.

Transport and land use networks mutually influence each other through the amount of accessibility the former allows and the resulting activities that can take place in the latter [16]. They are both determined by and determine the spatial configurations of the environment they are in (Figure 1, centre). Regarding transportation networks (see Figure 1, top), the classic network dilemma plays a role in understanding urban form and its spatial configuration [14,17-19]. An expanded network (i.e., streets or rail) consisting of many nodes and hubs and links and connections that obey a hierarchy developed over time [20]. Such a network might cover more territory and serve a larger user group and be more resilient [17]. However, the more complex and complicated the network becomes, the higher the costs are of utilising and maintaining such a network. For example, as the degree of transfers needed to get from A to B increases, so does time delays and energy usage [6]. The challenge here is therefore to strike for a balance between transport accessibility and sustainability at both the micro- and macroscale (see Figure 1, left and right). As for land use patterns (see Figure 1, bottom), density and diversity (mixed land use) are critical factors for sustainable outcomes [12]. The general understanding is that higher density urban environments will lead to less energy consumption. These factors can, 
however be relative to the context such as building typology and average household size [5,6]. It is therefore important to consider the socioeconomic effects of urban spatial configuration on land use in the form of activities available and how accessible this is for various demographic groups [16].

Critics say that although parts of this relationship (density and trip length) seem straightforward, other factors remain obscure [11]. Those factors tend to concern choice, particularly that of individual mobility choice or residential location choice [21]. However, these factors will not be modelled directly due to the fact of our focus on spatial configurations of the street and road network. Travel behaviour choice (i.e., route and mode) determined spatially could be derived from the spatial configuration analysis derived from the space syntax approach $[8,9]$, whereby the higher spatial integration of the network, the higher flow of movement. This is where we apply the space syntax method for identifying the spatial parameters in relation to transport energy consumption.

\subsection{Understanding Spatial Configurations with Space Syntax}

Methods for describing and measuring urban space stem from an analytical understanding of physical components developed in the 1950s amongst urban morphologists [22]. However, methods for analysing spatial configuration-space syntax — defined as the spaces between the physical objects have been consistently developed since the 1970s [8,9]. This method [8,23-27] helps in the development of precise definitions of the spatial elements focusing on extrinsic properties of space in terms of pure spatial relationships, and in developing theories on space and spatial relationships, on space and flow of movement, and economic attractiveness. Texture, shapes and form of the built environment is not at issue here. With current technology and software development and computer capacities, application of this analysis on large metropolises has become possible and widely utilised worldwide by an international community resulting in a substantial database confirming the method and its findings [28].

Space syntax consists of four theories: (i) the theory of natural movement [23], (ii) the theory of the natural movement economic process [24,29], (iii) theories of spatial combinatorics [8]; and (iv) the theory of the natural urban transformation process [29-31] that describe and predict effects of spatial configuration. The theory of the natural movement states that the flow of human movement in built environments depends on the degree of spatial integration (i.e., normalised distance from one point to all points [9]) of the street network. The higher spatial integration on various scale levels, the higher the flow of human movement [32]. The theory of the natural movement economic process states that the spatial configuration of the street network influences the flow of human movement and thereby the type, number, and location of shops $[24,29,32]$. The theory of the spatial combinatorics shows how an object placed in space contributes to integrate or to segregate urban spaces [8]. The theory of the natural urban transformation process shows that the spatial configuration of the street network steers building density and the degree of land use diversity in urban areas [29-31].

As research has shown, the spatial configuration of the street network is the underlying driving force for the densification processes of the built mass, the degree of land use diversity, the degree of movement flows through the street and road networks, and the dispersal of economic/commercial activities [27]. These theories allow us to calculate spatial inter-relationships based on three types of distance: topological (the number of direction changes), geometrical (the number of angular deviation) and metrical (the travelled distance), and correlate resulting spatial data with place bounded socioeconomic data (such as movement flow, property prices, distribution of crime, human behaviour in urban space etc.) $[26,27,29,30]$.

The measures within space syntax that best capture spatial configurations are [8,9];

- Potential to-movement: how likely a street is to be a destination of a route. Angular segment integration analyses (i.e., how close each segment is to all others in terms of the sum of angular changes that are made on each route); 
- Potential through-movement: how likely a street is used as part of a route. Angular choice analysis (i.e., counting the number of times each street segment falls on the shortest path-least angular deviation—-between all pairs of segments within a selected distance or radius).

In any urban environment, considering only spatial configuration and, ceteris paribus, route choice is determined primarily by, to and through movement. The other choice factor at play would be then mode choice, which is affected mainly by transport supply (i.e., if certain networks per modality are available), and resulting travel distances and travel times. Although these choices are subjective, both are factors in how transport energy usage is related to spatial configuration $[6,7,10,11]$. Therefore, by analysing spatial configurations and correlating it to transport energy consumption, the sustainability of urban forms can be assessed. The following section will deal with what inputs and variables are needed to implement such an assessment.

\section{Methodology}

To examine how and which spatial configurations affect energy usage (transport) in cities, this article proposed an integrated assessment method based on quantitative data on two levels of scale simultaneously (i.e., micro and macro). Two avenues of data and corresponding methods are needed to combine spatial configuration data analyses with space syntax and energy usage data via MATSim.

\subsection{Cases: Bergen and Zürich}

Two developed European cities with (i) dense transport networks (streets and transit), (ii) dense urban spatial configurations and (iii) situated in valleys between geological formations were selected due to the fact of their anticipated similar urban settlement patterns (see Figure 2).

The city of Bergen, located in western Norway, has a dense public transport network in its city centre. However, coverage decreases sharply after a couple of kilometres. Thereafter, there is a high dependency on private vehicle usage. This is reflected in its modal split (car- $55 \%$, public transport-16\%, cycling-3\% and walking-26\%) [33]. The most used public transport mode is the bus, followed by the newly established light rail system which runs through the valley leading away from the city centre. A second light rail line is expected by 2023.

The city of Zürich, in north-central Switzerland, is well known for its dense public transport network consisting of a wide range of modes that operate in tune with each other: trams, (trolleys) buses, local, regional and (inter)national trains, even a few boat services over the lake and funicular trains leading up the hills. This is reflected in its modal split (car-25\%, public transport- $41 \%$, cycling — $8 \%$ and walking—-26\%) [34].

Level of Scale

A radius $(\mathrm{R})$ of $500 \mathrm{~m}$ was taken for the local or microscale, and $5000 \mathrm{~m}$ for the macro or city scale for understanding urban spatial configurations. These distances are pertinent to how easy it is to get to a given location and how likely one is to pass through within certain metrical radiuses. These distances are applicable to the north-western European context [35] (p. 269). The above choices take the angular deviation of each topological step into account. The first radius concerns walkable distances or local accessibility (i.e., walking around 5-6 $\mathrm{min}$ at an average speed of $5 \mathrm{~km} / \mathrm{h}$ ), whereas the latter one concerns car-based distances or city scale accessibility (i.e., driving around 5-6 $\mathrm{min}$ at an average speed of $50 \mathrm{~km} / \mathrm{h}$ ). 


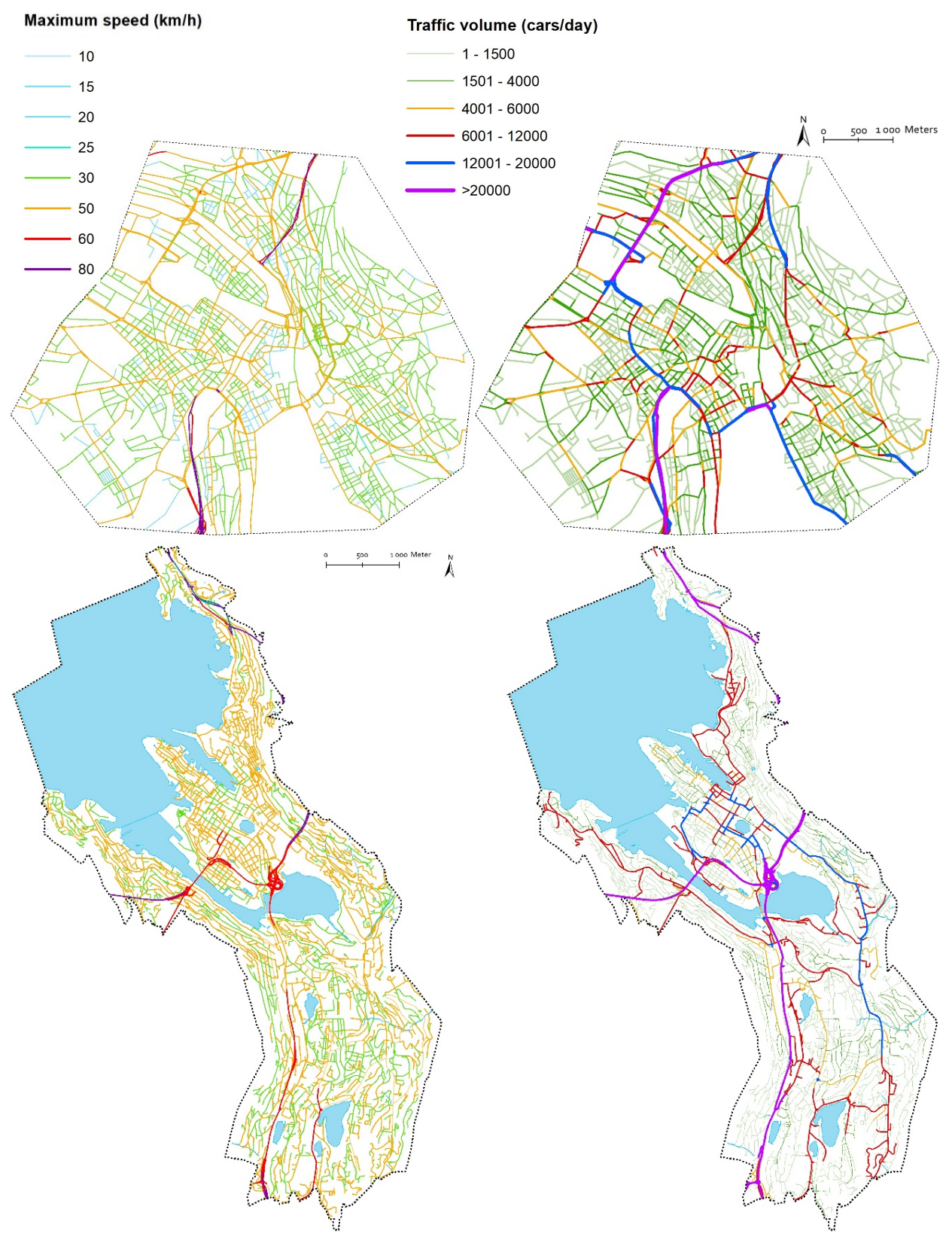

Figure 2. (left) Maximum speed in $\mathrm{km} / \mathrm{h}$ and (right) traffic volume in cars/day for networks in Zürich (top) and Bergen (bottom). Note that the purple, red and blue colours show the highest values for maximum traffic speed and the amounts of private vehicles, respectively. As can be seen for both Zurich and Bergen, the largest traffic flow of vehicle transport takes place on the roads with the highest speed. These are the motorways connecting the centres with the peripheral areas.

\subsection{Variables for Analysis}

Understanding that spatial configuration relies on extrinsic properties of space (i.e., structure of street networks) and no meaning is attributed to the built form [36], a combination of macroand microscale variables was used for analysis (see Table 1). Utilizing the most recent and novel variables of segment integration and angular choice analysis, the topological, geometrical, and metrical properties were considered in calculating spatial configurative relationships of street segments [28]. These variables were selected to best capture potential to movement (integration) and potential through movement (choice) to capture spatial configuration and to reflect transport energy consumption. 
Table 1. List of variables used in the model.

\begin{tabular}{|c|c|c|c|}
\hline Variable & Scale & Description & Metric \\
\hline Segment Integration $(\mathrm{R}=500 \mathrm{~m})$ & Micro & $\begin{array}{l}\text { To-movement potential with } \\
500 \mathrm{~m} \text { metric radius }\end{array}$ & Numeric \\
\hline Angular Choice $(\mathrm{R}=500 \mathrm{~m})$ & Micro & $\begin{array}{l}\text { Through-movement potential } \\
\text { with } 500 \mathrm{~m} \text { metric radius }\end{array}$ & Numeric \\
\hline Segment Integration $(\mathrm{R}=5000 \mathrm{~m})$ & Macro & $\begin{array}{l}\text { To-movement potential with } \\
5000 \mathrm{~m} \text { metric radius }\end{array}$ & Numeric \\
\hline Angular Choice $(\mathrm{R}=5000 \mathrm{~m})$ & Macro & $\begin{array}{l}\text { Through-movement potential } \\
\text { with } 5000 \mathrm{~m} \text { metric radius }\end{array}$ & Numeric \\
\hline Aggregated Angular Choice & $\begin{array}{l}\text { Micro/ } \\
\text { Macro }\end{array}$ & $\begin{array}{l}\text { Combination of high and low } \\
\text { radius }([\mathrm{C} 500] \times[\mathrm{C} 5000])\end{array}$ & Numeric \\
\hline Energy consumed without stops & Macro & $\begin{array}{c}\text { Total car energy usage per } \\
\text { street segment per day } \\
(\mathrm{kWh} / \text { day })\end{array}$ & Numeric \\
\hline
\end{tabular}

\subsection{Data Source and Inputs}

To fulfil the list of variables, the following data sources were then prepared and transformed for analysis depending on the respective platform for the necessary list of variables (see Table 2).

Table 2. Data source, input form, and platform.

\begin{tabular}{|c|c|c|c|}
\hline Data Source & Input form & Platform & Outcomes \\
\hline OpenStreetMap & Axial Map & DepthMap & $\begin{array}{l}\text { Potential to-movement with } \\
\text { Angular Choice (Low/High) }\end{array}$ \\
\hline OpenStreetMap & Axial Map & DepthMap & $\begin{array}{c}\text { Potential through movement } \\
\text { with Angular } \\
\text { Integration (Low/High) }\end{array}$ \\
\hline $\begin{array}{l}\text { OpenStreetMap } \\
\text { (Zürich case) }\end{array}$ & $\begin{array}{l}\text { Traffic speed and vehicle } \\
\text { usage derived from } \\
\text { polyline-edges in network }\end{array}$ & MATSim & $\begin{array}{c}\text { Transport energy consumed } \\
\text { (Changing Speed, }\end{array}$ \\
\hline $\begin{array}{l}\text { Road data from } \\
\text { Norwegian Road } \\
\text { Authorities } \\
\text { (Bergen case) }\end{array}$ & Node/Link Network & GIS & $\begin{array}{l}\text { Air Resistance, Rolling } \\
\text { Resistance and Heat) }\end{array}$ \\
\hline
\end{tabular}

\subsection{Variables for Spatial Configuration}

The spatial structure of the street network forms the basis of the space Syntax analyses. The data of street networks per case were sourced from OpenStreetMap (OSM) between April-June 2016 for Bergen and July-September 2017 for Zürich. Georeferenced, axial maps were drawn during the same period and confirmed using a combination of Google Street View and OSM road centre line data using ArcMap. The axial maps follow the principle that the fewest and longest set of axial sight lines of visibility and accessibility cover all convex spaces in a spatial system [28] (p. 13). It provides the empirical data for calculating segment integration (to-movement), angular choice (through-movement) at both scales, and aggregated angular choice is derived from buffers on each segment.

\subsubsection{Segment Integration (Potential To-Movement)}

With the input of the axial map, DepthMap is used to calculate the topological depth from one axis to all others-the total number of direction changes from one axis to all others. The integration (I) of an axial line (i) is a function of its depth related to all other axes calculated as [37] (p. 64):

$$
I_{i}=\frac{2\left(\mathrm{n}\left(\log _{2}\left(\frac{n+2}{3}\right)-1\right)+1\right) /(n-1)(n-2)}{2\left(\left(\frac{\sum_{j=1}^{n} d_{i j}}{n-1}\right) /(n-2)\right.}
$$


where $n$ is the number of segments, $d_{i j}$ is the shortest distance (fewest number of direction changes) between two segments $i$ and $j$. The greater the number of steps $\left(\mathrm{d}_{\mathrm{ij}}\right)$ between streets axes, the lower the integration values gets.

Segment integration of a street shows how easy it is to get to a segment from all other segments. In the segment analyses, the axial lines are broken up where they cross each other [28]. It calculates the to-movement potentials. Segment integration can be compared across systems. It measures how close each segment is to all others in terms of the sum of angular changes that are made on each route [37] (pp. 475-490). Angular segment choice is calculated by counting the number of times each street segment falls on the shortest path between all pairs of segments within a selected distance (termed "radius"). The "shortest path" refers to the path of least angular deviation (namely, the straightest route) through the system [37] (p. 475).

The angular integration (AI) of a segment $\mathrm{x}$ is:

$$
A I_{x}=\frac{1}{n} \sum_{i=1}^{n} d_{\theta}(x, i)
$$

where $n$ is the number of segments and $\mathrm{d} \theta$ the angle between any two segments on the shortest path on a segment $x$ and when adding the length " $l$ " of segments [37] (p. 66):

$$
A I_{x}^{l}=\frac{\sum_{i=1}^{n} d_{\theta}(x, i) l(i)}{\sum_{i=1}^{n} l(i)}
$$

\subsubsection{Angular Choice (Potential Through-Movement)}

Angular choice, or how likely one is to pass through that axis when moving around in a built environment, measures the degree of betweenness and measures the through-movement potentials. The formula of angular choice (C) of an axis (i) is as follows [37] (p. 64):

$$
\mathrm{C}_{i}=\sum_{j} \sum_{k} g_{j k}^{(i)} / g_{j k}^{(j<k)}
$$

where $g j k(i)$ is the number of shortest paths between segment $j$ and $k$ containing $i$, and $g j k$ is the number of all shortest paths between $j$ and $k$.

\subsubsection{Aggregated Angular Choice}

Values generated by angular choice analyses are aggregated per case with a $35 \mathrm{~m}$ buffer around each segment, creating aggregated areas for each integration level. This value is based on various research concluding that a dense street network with a fine mesh size of between $60-80 \mathrm{~m}$ performs better than larger blocks, both when it comes to increased circulation and the exploitation possibilities of the urban block [28]. Showing angular choice for high and low radius simultaneously helps to find out which areas are well integrated into the local street network and enjoy good accessibility on the city scale. The natural break-or Jenks-method is applied to classify the resulting spatial values from angular choice as low $(\mathrm{L})$, medium $(\mathrm{M})$ or high $(\mathrm{H})$. This allows for a combination of nine aggregated choice categories that represent typologies of routes (see Table 3).

\subsection{Variables for Transport Energy Usage}

Focusing on energy usage for private vehicles, data were generated via MATSim—an agent-based program for making large-scale transportation simulations. Agents, representing residents, are assigned a home address and a job or study location. Daily activity schedules can be appointed or generated, after which the agents will choose a travel itinerary based on the transportation options available. The agents' route-choice and mode-choice between their origins and destinations are then made based 
on travel time and costs through an iterative optimisation process. The simulation accuracy depends on the amount of detail of the parameters programmed into the model [38].

Table 3. Aggregated choice categories matrix. High values have a dark shade, and colour red if angular choice values at city-wide scale (global) are higher than local scale choice values and green if local scale choice values are higher than city scale choice values.

\begin{tabular}{ccccc}
\hline & & \multicolumn{3}{c}{ Angular Choice with Low Radius (R = 500 m) } \\
\hline & & Low & Medium & High \\
Angular Choice & Low & LL & LM & LH \\
with High Radius & Medium & ML & MM & MH \\
$(\mathbf{R = 5 0 0 0 ~} \mathbf{)})$ & High & HL & HM & H \\
\hline
\end{tabular}

\subsubsection{Simulating Energy Usage}

In this paper, the choice to focus on energy data from private car use was due to the following reasons, (i) only accurate car traffic data could be obtained for both cities, (ii) public transport lines are subject to non-rational planning choices and do not always follow the most integrated, fastest or shortest routes, whereas private car drivers do, (iii) the current data availability for both cities made it is difficult to determine the itinerary of passengers, and therefore it was impossible to estimate how many trips a bus line represents. On the contrary, for cars, one trip was (assumed) equal to one. Since pedestrians and cyclists move around using energy they "produce themselves", the energy usage by cars is the one mode that is most practical for comparing energy usage with to-movement and through-movement potentials of spatial configurations. Agent-based simulations can assist in demonstrating a change in the agents' choice of mode of transportation, for example, if a change occurs in the public transport network or a change in the road network. In this article, the focus remained with private vehicles usage.

Energy consumption was calculated per street segment and relevant parameters giving information about the amounts of vehicles that used a specific street segment and how much energy each vehicle potentially consumed. For the Zürich analysis, data were generated through an agent-based simulation program (MATsim). This input data were maximum traffic speed (see Figure 2, left) and amount of (private) vehicles observed (see Figure 2, right). For the Bergen analysis, the actual data were sourced from the national road authorities.

The following calculations were used to estimate the amount of energy usage per vehicle $\left(\mathrm{E}_{\mathrm{car}}\right)$ from one point to another: kinetic energy in changing speed (and direction), air (swirl) resistance ( $\left.E_{\text {air }}\right)$, rolling resistance $\left(\mathrm{E}_{\text {roll }}\right)$ and heat loss $\left(\mathrm{E}_{\text {heat }}\right)$. Without the amount of energy that the industry needs to produce the car and the fuel itself, the total amount of energy that a driving car's engine produces was converted to other forms of energy under the influence of the following [38]:

\subsubsection{Changing Speed (and Direction)}

After a vehicle with mass $m$ speeds up to a velocity $v$, the built-up kinetic energy is converted by the brakes into heat at stopping points such as traffic lights and pedestrian crossings. Kinetic energy is calculated by the formula:

$$
\mathrm{E}_{k}=\frac{1}{2} m v^{2}
$$

The problem with the parameter for distance is that the way the segments are split up in the model, this formula would assume that each car comes to a full stop at the end of each street segment. The model, thus far, lacks the data on stopping points. However, it is also not possible to calculate the aggregated losses from, amongst others, subtler braking, taking turns, and sloped terrain accurately. To eliminate this inaccuracy, the kinetic energy for braking is left out of the equation. The final estimation of energy usage will therefore be modest, at best, compared to the actual numbers. 


\subsubsection{Air Resistance}

The swirl of air around the car causes a drag, the coefficient of which, c, is depending on the cross-sectional area, size and shape of the vehicle. Following MacKay [39], we here assumed an average car drag value of 0.33 . The effective area $\mathrm{A}_{\mathrm{air}}$ of the air swirl (resistance) was calculated by multiplying the cross-sectional area $\mathrm{A}_{\mathrm{car}}$ of the vehicle by this drag-coefficient:

$$
\mathrm{A}_{\text {air }}=\mathrm{c} \cdot A_{\text {car }}
$$

For air resistance, the kinetic energy of the swirl of the air was calculated. The mass is found by multiplying the density by volume. The volume of the tube of air was obtained by multiplying the effective area A by the length of the tube, obtained by vt. The mass of the tube of air is then:

$$
\text { Mass }=\text { density } \cdot \text { volume }=\rho \text { Avt }
$$

where $\rho$ is the density of air, which is $1.3 \mathrm{~kg} / \mathrm{m}^{3}$ at sea level. The kinetic energy of the air swirl is then:

$$
\mathrm{E}_{\mathrm{air}}=\frac{1}{2} \mathrm{~m}_{\mathrm{air}} \mathrm{v}^{2}=\frac{1}{2} \rho \mathrm{Avtv}^{2}
$$

which per time unit comes down to:

$$
\frac{\rho A v t v^{2}}{t}=\rho A v^{3}
$$

\subsubsection{Rolling Resistance}

This is a constant coefficient that depends on the vehicle's mass and is typically 0.01 for cars. A $1000 \mathrm{~kg}$ car with a 0.01 rolling resistance coefficient then requires $0.01 \cdot 1000=100 \mathrm{~N}$. With: power $=$ force $\cdot$ velocity, rolling resistance is directly related to the speed in $\mathrm{m} / \mathrm{s}$ by a factor 100 :

$$
\mathrm{E}_{\text {roll }}=100 \mathrm{v}
$$

\subsubsection{Heat}

The poor energy-converting capabilities of conventional fossil-fuel engine cars makes that approximately three-quarters of energy which is lost to heat. Whilst modern cars are fortunately becoming increasingly efficient, a factor of 4 is usually assigned to car engine heat loss. So, when we count with $75 \%$ heat loss, an average mass of a car of $1000 \mathrm{~kg}$ and an average cross-sectional area of $2.4 \mathrm{~m}^{2}$, the formula for the total amount of energy $\left(\mathrm{E}_{\mathrm{car}}\right)$ consumed by one driving car is:

$$
\begin{gathered}
\mathrm{E}_{\mathrm{car}}=\left(\mathrm{E}_{\text {air }}+\mathrm{E}_{\text {roll })} \cdot \mathrm{E}_{\text {heat }}\right. \\
=\left(\frac{1}{2} \rho A \mathrm{v}^{3}+100 \mathrm{v}\right) \cdot 4 \\
=4 \cdot\left(\frac{1}{2} \cdot 1.3 \cdot 0.8 \cdot \mathrm{v}^{3}+100 \mathrm{v}\right) \\
=4 \cdot\left(0.52 \mathrm{v}^{3}+100 \mathrm{v}\right) \\
=2.08 \mathrm{v}^{3}+400 \mathrm{v}
\end{gathered}
$$

Now, we can generate results per street segment. The total amount of energy $E_{\text {tot }}$ used by a given number of vehicles that drives through a certain street per day at a certain speed $(v)$ is:

$$
\mathrm{E}_{\mathrm{tot}}=(\text { number of cars per day }) \cdot\left(2.08 \cdot(\text { traffic speed })^{3}+400 \cdot(\text { traffic speed })\right)
$$

The above energy usage results are then linked with the georeferenced street segments with traffic speed as inputs as represented in Figure 2. 


\subsection{Integrated Assessment Approach}

The integrated assessment approach consists of three cascading steps:

1. Generating aggregated angular choice buffers resulting in route typologies (see Table 3, Figure 7, left and Figure 8, left);

2. Spatial configuration and energy-use overlay (see Figures 3-6) for to- and through-movement;

3. Testing for co-relation between spatial configuration variables (see Table 1).

Step 1 was done by grouping the values of angular choice for each scale level into three categories: low (L), medium (M) and high $(\mathrm{H})$ using the natural break method, creating aggregated areas for each category by generating a $35 \mathrm{~m}$ buffer around the segments.

Step 2 aggregated the categorised angular choice values $(A C)$ on the high $(R=5000)$ and low $(R=500)$ radius by permutation as shown in Table 3 corresponding to the buffers created in Step 1. The high local values are emphasised in red and local values in green.

Step 3 was achieved by spatially overlaying the layer containing the energy values and subsequently running a bivariate analysis of the dependent variable for energy (energy consumed, $E_{\text {tot }}$ ) and the independent spatial configuration variables of the aggregated segment values of angular choice (AC) on both the micro- and macroscales.

Steps 1-3 allows for analysis of the spatial configuration's relationship to energy usage and allows us to identify types of routes that might be present. This allows for statistical and visual-spatial understanding of that relationship on both micro- and macro-scale levels. Step 3 in particular provides typologies which can further inform planning policy and practice. These outcomes are demonstrated in the findings for both cases.
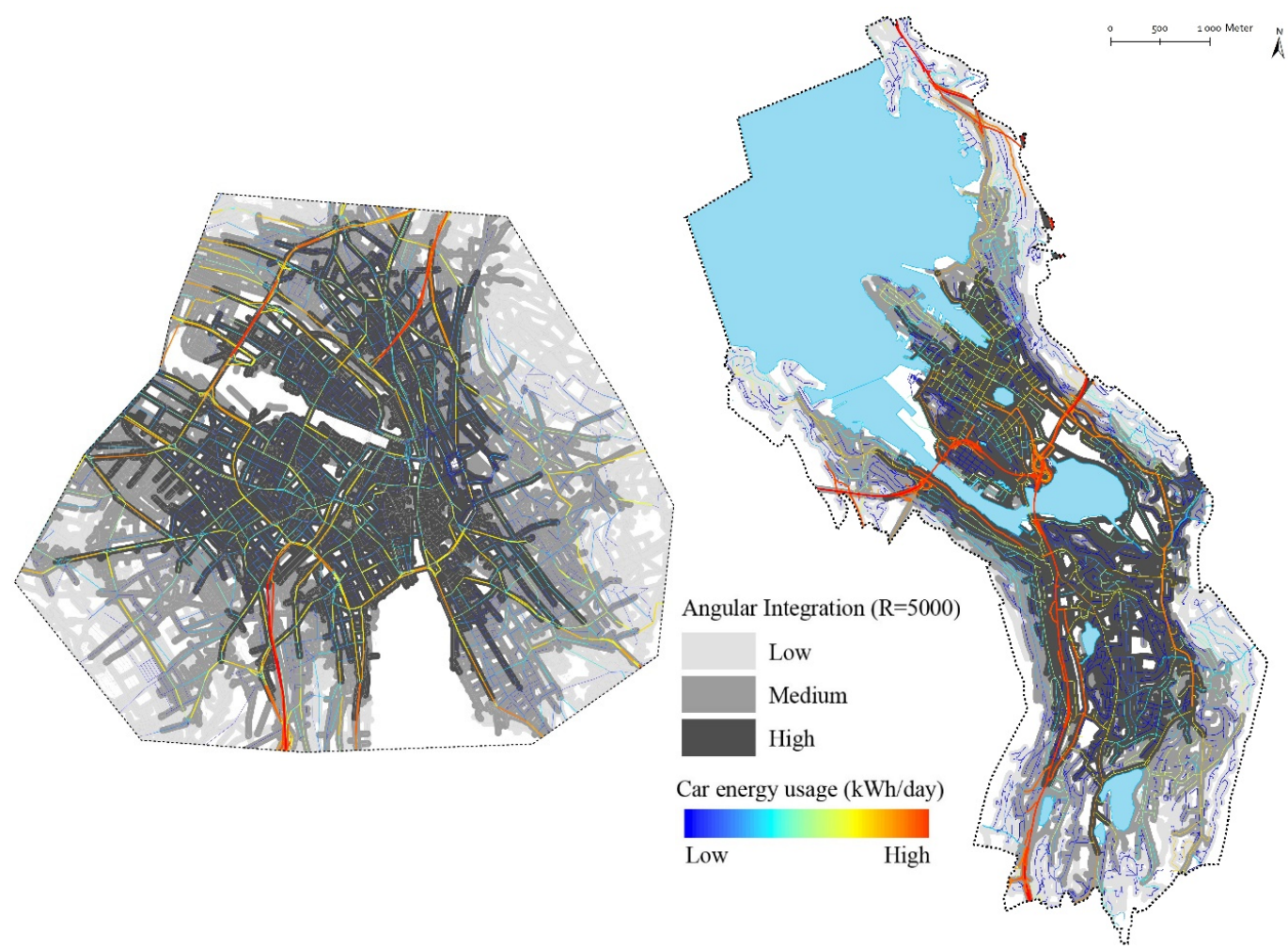

Figure 3. Energy usage from cars overlapped on segment integration analyses with a high metrical radius $(\mathrm{R}=5000 \mathrm{~m})$. 

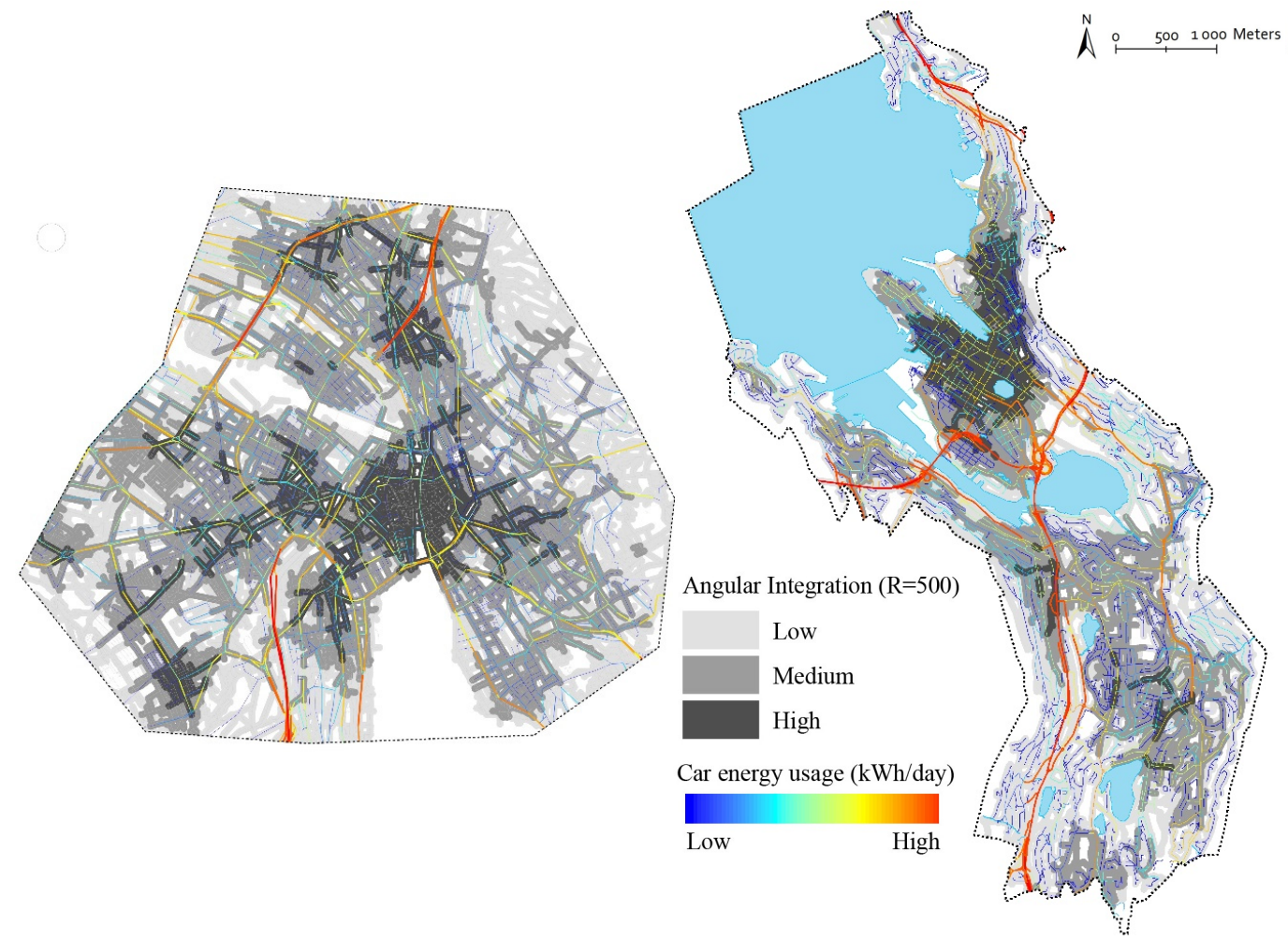

Figure 4. Energy usage from cars overlapped on segment integration analyses with a low metrical radius.
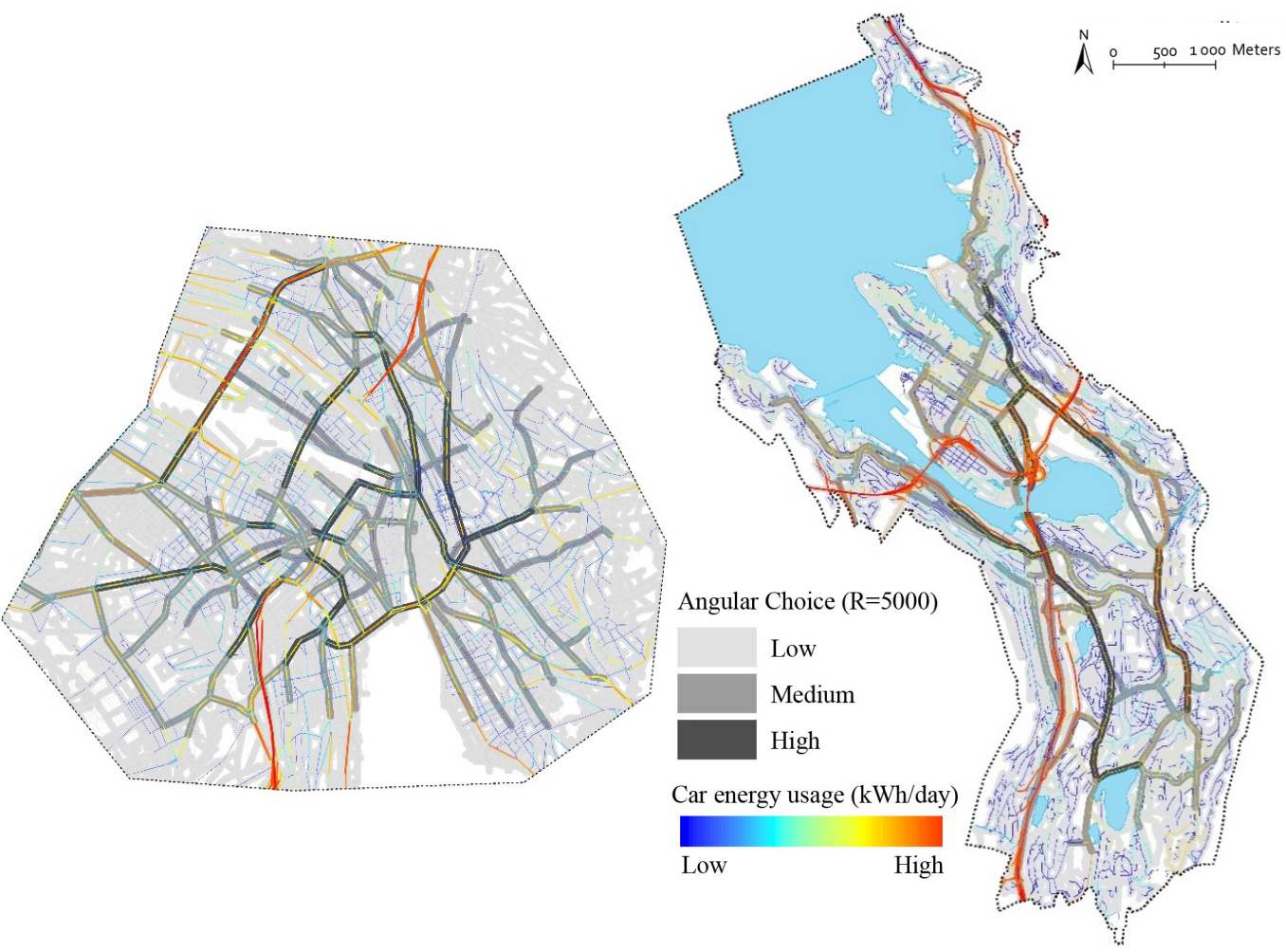

Figure 5. Energy usage from cars overlapped on angular choice analyses with a high metrical radius at city scale. 

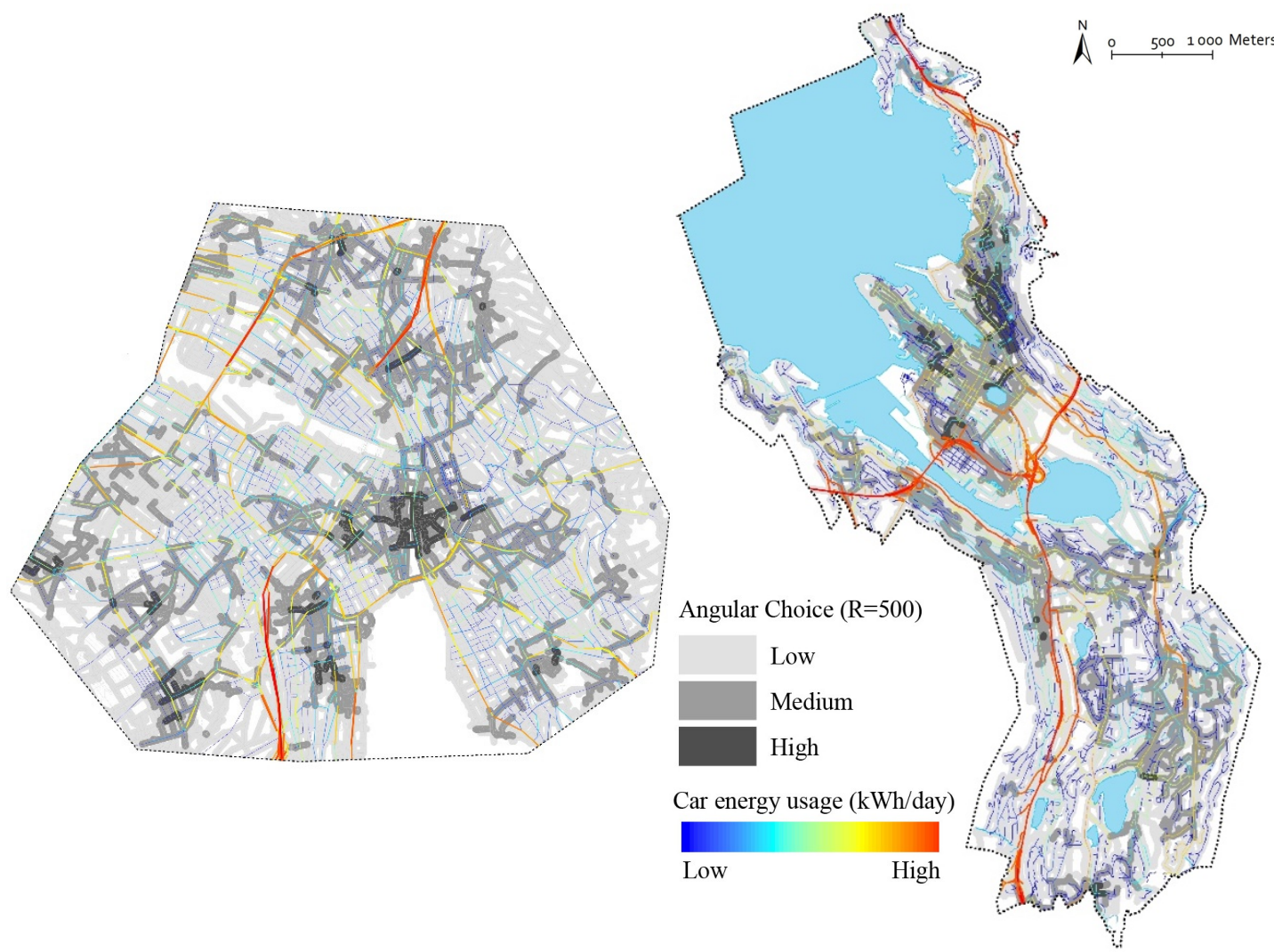

Figure 6. Energy usage from cars overlapped on angular choice analyses with a low metrical radius at local scale.

\section{Findings}

Statistical correlation between spatial configurations and transport energy usage indicate a positive relationship between all variables for Zürich and Bergen with the exception of segment integration at local scale for Bergen (see Table 4). When comparing the aggregate angular choice of both cities, segments with high values for local integration (i.e., walkable areas) tend to use less energy than areas with high values at a city-wide scale (i.e., car-oriented). The following sections will discuss these key findings in detail before discussing the typologies of routes from these findings.

Table 4. Relating spatial configuration variables (segment integration and angular choice) at microand macroscale to transport energy usage.

\begin{tabular}{ccccccccc}
\hline \multicolumn{4}{c}{ Bergen } & \multicolumn{2}{c}{ Zurich $^{*}$} \\
\hline & \multicolumn{2}{c}{ Sample } & \multicolumn{2}{c}{$N=2174$} & \multicolumn{2}{c}{ Sample } & \multicolumn{2}{c}{$N=7027$} \\
\hline Analysis & \multicolumn{2}{c}{ Segment Integration } & \multicolumn{2}{c}{ Angular Choice } & \multicolumn{2}{c}{ Segment Integration } & Angular Choice \\
\hline Radius $(m)$ & 500 & 5000 & 500 & 5000 & 500 & 5000 & 500 & 5000 \\
\hline $\begin{array}{c}\text { Pearson } \\
\text { Correlation }\end{array}$ & $\mathbf{- 0 . 0 6 1 * *}$ & $0.129 * *$ & $0.053^{*}$ & $0.149 * *$ & $0.079 * *$ & $0.113^{* *}$ & $0.024^{*}$ & $0.073^{* *}$ \\
Significance & 0.005 & 0.000 & 0.013 & 0.000 & 0.000 & 0.000 & 0.047 & 0.000 \\
\hline
\end{tabular}

* Correlation is significant at the 0.05 level (two-tailed). ${ }^{* *}$ Correlation is significant at the 0.01 level (two-tailed).

\# Outliers due to the motorway removed ( $10 \%$ of energy consumption). -0.061 is an outlier value

\subsection{Correlating Spatial Configurations to Transport Energy Usage}

Spatial configuration analysis generated a database for potential to- (segment integration) and through-movement (angular choice) per segment for both cases. Segment size differ between Bergen $(N=2174)$ and Zurich $(N=7027)$ as evidenced by the different city forms (see Figure 3$)$. Table 4 shows 
the correlation between energy usage for transport and segment integration and angular choice with both low $(R=500$, micro scale) and high $(R=500$, macro scale) metrical radius for Bergen and Zürich. All correlations are significant for both cases. Positive correlations were found between spatial configuration variables and energy usage for transport except for integration at low metrical radius (local scale) for Bergen.

- In terms of to-movement (i.e., how likely a street is a destination, measured through Angular Integration), Bergen shows a negative correlation at the local or micro scale but a positive correlation at the city-wide or macroscale. This means that in Bergen, the less a street is a walkable destination (lower integration value), the higher the transport energy use. Whereas in streets that are accessible destinations for vehicular traffic (integration at macro scale), a higher transport energy use is expected;

- In terms of to-movement for Zürich, for both the micro- and macro-scale, a positive correlation for segment integration was observed. This means that in Zürich, the more walkable destinations (the higher the choice value), the higher transport energy use was observed, contrary to literature $[10,11,40]$. A possible explanation for this may be the difference between car usage in Bergen (55\%) and Zürich (25\%) [33,34]. Having relatively low care ownership in Zürich compared to Bergen may exacerbate the share of local traffic in the agent-based simulation when correlating with the segment values. In addition, Zürich's pedestrian zones (streets such as Augustinergasse) are not completely blocked off and still accommodate private vehicles or goods delivery vehicles;

- In terms of through-movement (i.e., how likely a street is a part of route, measured through Angular Choice), Bergen and Zürich both showed a positive, albeit weak, significant correlation on both scale levels. Therein, the more a street was likely to be part of a route choice (the higher the angular choice value), the higher the energy use for transport was expected. This was more so for the city scale than the local scale. Both cities have limited, continuous pedestrianized zones (within a one-kilometre radius) where cars are limited. This might affect the segments with high angular choice values that could be dominant, prioritised, and potentially historical routes in the city as they naturally become extensions into major roads [20,41]. Next, the adjacent areas to these pedestrian zones tend to have walking pavements parallel to roads allowing vehicular traffic. Even though these routes are likely to be chosen, they facilitate both pedestrian and vehicular traffic simultaneously.

\subsubsection{Segment Integration (To-Movement or Destination)}

If transport energy use, the degree of land use diversity and building density depend on the spatial configurations of the street and road networks, then it follows that areas where the largest function mixture and the largest building density is present, less transport energy is consumed. In Zürich centre, both walkability (26\%) and public transport (41\%) usage were high [34]. In Figure 3 we see that as soon as the integration values drop (dark grey to light grey) towards the edge of the inner city, the energy usage for transport increases (orange and red). This is even more observable in areas that enjoy a high integration values at the city scale, but low integration values at the local scale as seen in comparing Figures 3 and 4 .

\subsubsection{Angular Choice (Through-Movement or Route Choice)}

Streets segments that have a high angular choice value at the local scale and a high transport energy consumption, also have high or medium angular choice values at the city scale. It is clearly visible in how the "aortae" of high energy usage "feed" and connect the areas of high local through-movement (see Figures 5 and 6). This corresponds to path dependencies of cities in which historically important routes retain a high level of use and recognisability for the population and eventually evolve into major traffic thoroughfares that facilitate private vehicle usage [41]. 


\subsubsection{Aggregated Angular Choice}

Figure 7 (left) is a representation of aggregated and buffered choice values on both the local and the city-wide scale for Bergen. High values have a dark shade, and colour red if angular choice values at city-wide scale (global) are higher than local scale choice values and green if local scale choice values are higher than city scale choice values. Correlating the maps in Figure 7 (left) and (right), areas with high or medium local scale values present lower energy usage. High values at the city scale present higher energy usage. Areas with high global and low local angular choice values (HL) (bright red) scores marginally higher in energy usage than medium global and medium local (MM) (dark grey) integration. It seems that high angular choice values for local scale have a bigger impact on energy reduction than global values in Bergen.
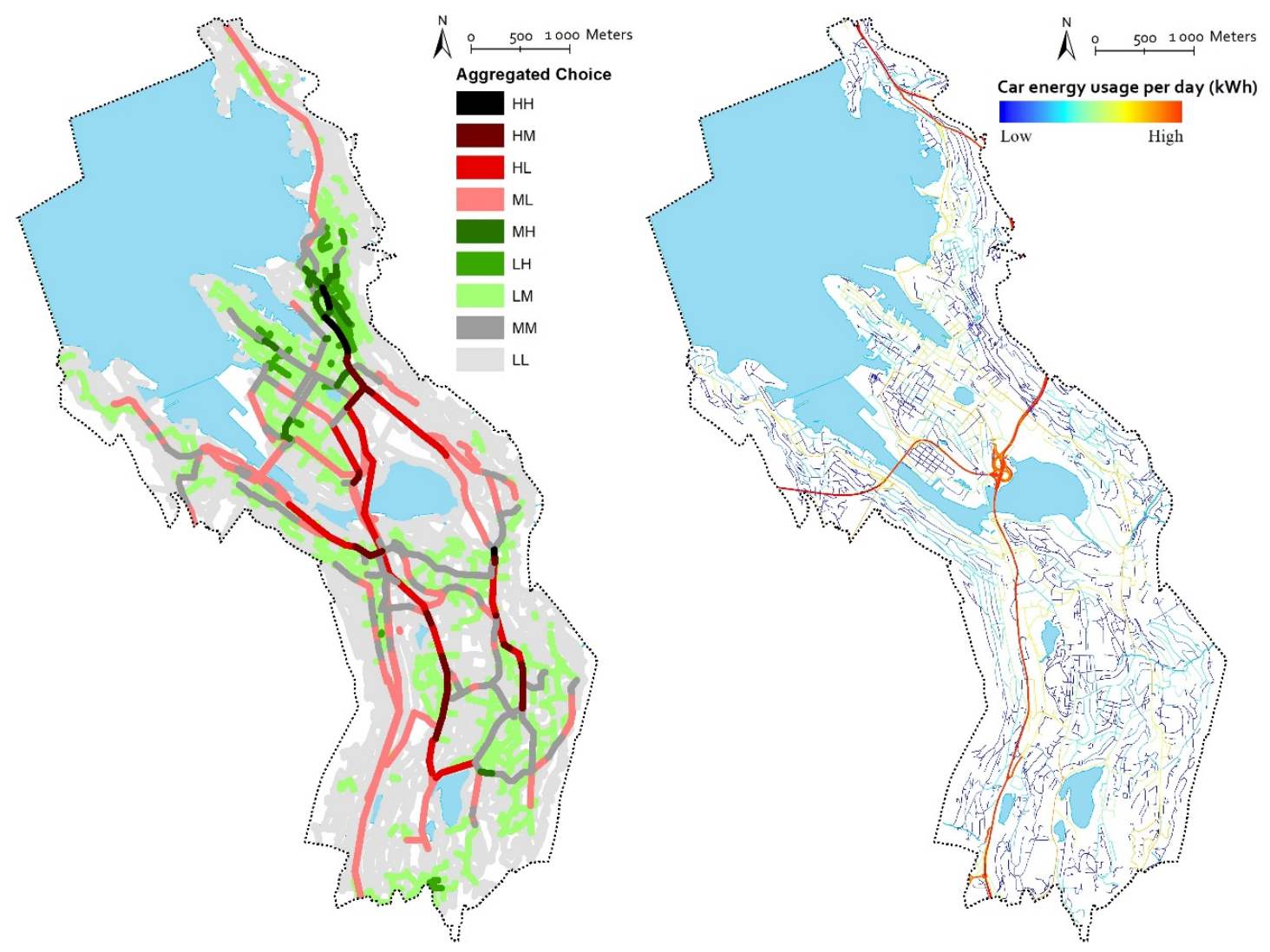

Figure 7. Aggregated angular choice (left) and energy usage for cars (right) in Bergen.

Figure 8 (left) is a representation of aggregated and buffered choice values on both the local and the city wide (global) scale for Zürich. Correlating with energy use represented in Figure 8 (right) and the spatial configuration (Figure 8, left), it shows a similar pattern in Zürich as for Bergen. It can be observed that areas with high or medium local values score lowest in energy usage. In Zürich, segments with low values at global scale and medium values at local scale (LM) show a higher energy usage than those with medium values at global scale and low values at local scale (ML). Likewise, segments with high values at global scale and medium values at local scale (HM) has higher energy usage values than those with high values at global scale and low value at local scale (HL). 


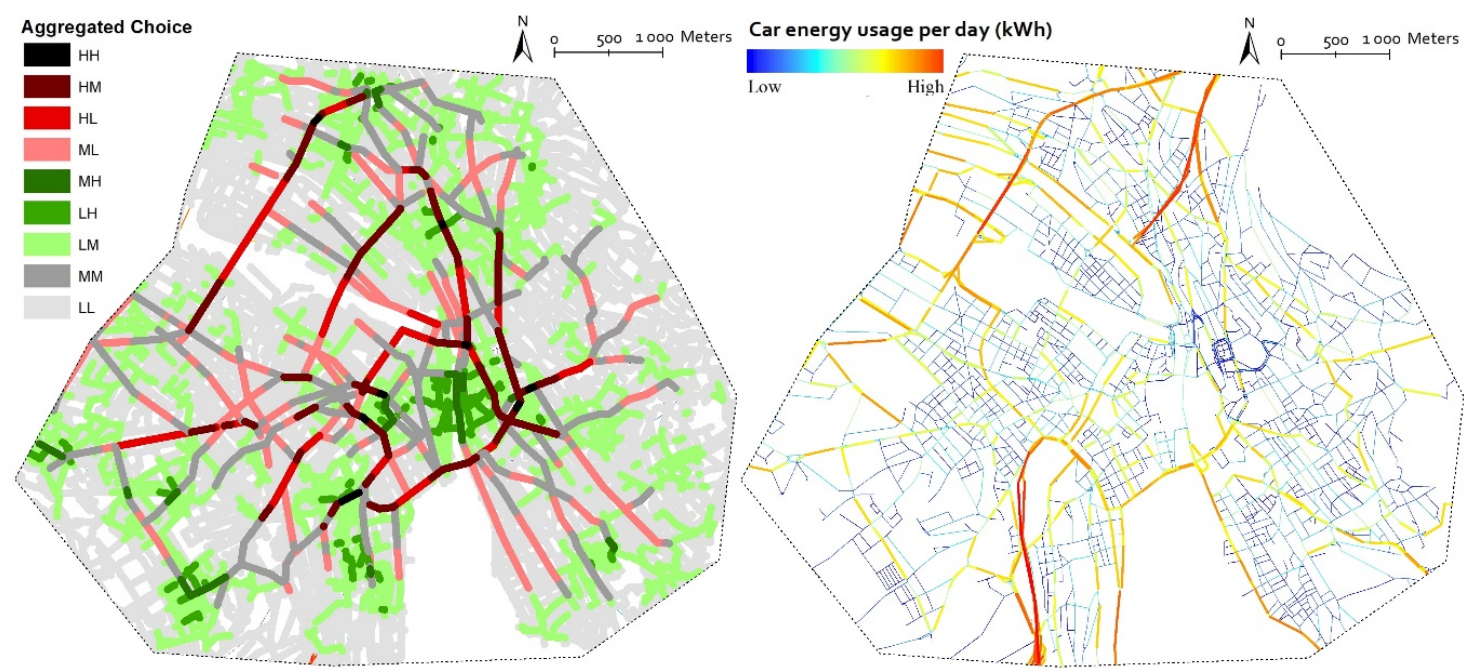

Figure 8. Aggregated angular choice (left) and energy usage for cars (right) in Zürich.

The similarities between both cities are however evident: all areas with high local integration, $(\mathrm{LH}),(\mathrm{MH})$ and $(\mathrm{HH})$ present as lowest in energy usage. Areas with low local integration values tended to present as high in energy usage.

\subsection{Identifying and Understanding Route Typologies}

Knowing the local and city-wide scale integration and choice values of the cities in relation to their energy usage provides the opportunity to relate this to typical transport and land use typologies. Interpreting the results from correlating spatial configurations and energy usage, the resulting typologies can be identified:

- High global, high local choice values (HH): Major road-Connects city districts, often supporting high volumes of traffic. When possible also used intensely by local pedestrians and cyclists;

- High global, medium local choice values (HM): Central road connecting city districts and the wider region; supports high volumes of regional traffic, and moderate local traffic;

- High global, low local choice values (HL): Regional road, often a motorway or boulevard; supports high speed, large volumes of traffic; little to no local traffic;

- Medium global, high local choice values (MH): District road, connecting neighbourhoods; moderate to high traffic volume, intensely used by pedestrians and cyclists;

- Medium global, medium local choice values (MM): District or local street that supports moderate traffic, often a mix of motorised traffic, pedestrians and cyclists;

- Medium global, low local choice values (ML): District or local road, predominantly for local motorised traffic travelling within and in between neighbourhoods;

- Low global, High local values (LH): Central street within or in between neighbourhoods; high intensity of local traffic, often non-motorised;

- Low global, medium local values (LM): Neighbourhood street with mixed functions, moderate traffic intensity, mostly for local residents;

- Low global, low local values (LL): Local road or street serving only the immediate surrounding properties.

This interpretation allows for an understanding of what these typologies in relation to spatial configurational values may represent in reality. However, since the analysis of spatial configuration merely describes the extrinsic properties of space these typologies still need to be controlled in the field. For example, in Bergen some of the most highly integrated segments (HH) (e.g., Stølegaten, Øvregaten), are in fact narrow which do not support large volumes of vehicular traffic. Conversely, some of the 
most spatially segregated segments (LL) experience heavy traffic (e.g., Sjøgaten, Møllendalsveien) and could be better typified as district roads (MM/ML). This discrepancy is useful for planning policies and strategies as it indicates the potential that a segment might not match or complement its current usage pattern. This would be helpful for planners to identify routes that might need upgrading (increase in capacity) or change in function (car-oriented to pedestrian-oriented).

\section{Conclusions}

To understand how spatial configurations can affect transport energy usage in cities, this article correlated six spatial and energy-usage variables (see Table 1) across the city of Bergen and Zürich. The values of integration (to-movement) and route choice (through-movement) at both the local and city-wide scales were determined using space syntax analyses of axial maps of both cities based on OpenStreetMap. This was then correlated statistically and spatially to transport energy-usage derived from data from the local municipality in Bergen and an agent-based model for Zürich based on traffic speed and volume.

\subsection{Spatial Configurations Affecting Transport Energy Usage}

Our findings indicate that the spatial structure of urban space affects transport energy usage. In general, there were weak but positive correlations to transport usage between segment integration and angular choice values at both local (low metrical radius, $\mathrm{R}=500 \mathrm{~m}$ ) and city-wide (high metrical radius, $R=5000 \mathrm{~m}$ ) scales for both cities (see Table 1 ). The correlation was stronger for both integration and choice at the city-wide scale, indicating that these routes which favoured vehicles tended to exhibit high transport energy usage. The exception here is that of segment integration at the local scale. Bergen showed this with a weak, negative relationship to transport energy usage. This indicates that the more "walkable" areas of Bergen showed less transport energy usage. Across both cities, correlation at the local scale was lower than correlation at the city-wide scale. This indicates that even though "walkable" or pedestrian-oriented areas (local scale) still exhibited a positive relationship to transport energy use, the coefficients were less than those of areas (city-wide scale) facilitating vehicles which are more car-oriented.

\subsubsection{High Transport Energy Usage for Car-Oriented Spatial Configurations}

The private car in particular is a major contributor to transport energy usage. As shown in the energy usage equation, longer and high-speed car trips consumed exponentially more energy. This is visible in Figure 3, where the high-energy usage areas were at the periphery of the medium-to-low values of segment integration at a city-wide scale. This is even more visible on a local scale (see Figure 4). These areas with less potential as destinations (to-movement), typically suburbs, urban peripheries or industrial zones, tended to see higher transport energy usage due to the higher car use or speeds permitted as opposed to denser city centres which might be pedestrianised or had lower speed limits. Examples include Mindemyren in Bergen and Industriequartier in Zürich. In Figure 5, the street segments with high angular choice values exhibit high transport energy usage. These arterial routes have the highest potential as chosen routes and attract high amounts of traffic and corresponding energy usage. Here, path dependencies might be at play as historically important routes tend to be routes that develop for vehicular traffic in modern times.

\subsubsection{Low Transport Energy Usage for Pedestrian-Oriented Spatial Configurations}

On a local scale (see Figure 6, the inverse is true. These segments with high angular choice at local scale tend to indicate a high degree of "walkability" in streets and attract less vehicular traffic and transport energy usage. Segments with high angular choice values at both scales but not high transport energy usage indicating an efficient public transport system for tram, buses or light rail commonly found in Bergen and in Zürich. Areas such as the peripheries of Byparken in Bergen and Altstadt in Zürich are where local scale high to-movement networks (segment integration) are enmeshed with 
the global scale high through-movement (angular choice) networks (see Figures 4 and 5). These areas reflect lower transport energy usage as private car usage is available but discouraged. Here, walking and cycling seem to become a natural choice for shorter, local destination trips. When these segments have the presence of a well-integrated and diverse public transport systems, these local destination trips have the potential to extend towards car-free regional trips, too, reducing energy usage further.

\subsection{Integrated Assessment for Transport and Land Use Planning}

This article proposed an integrated assessment approach consisting of three steps: (i) generation of aggregated angular choice buffers resulting in route typologies, (ii) spatial comparison of spatial configurations and energy usage via overlay and (iii) statistical correlation of spatial configurations and transport energy usage. Our findings indicated a clear correlation between spatial configurations and transport energy usage as depicted through nine common typologies. These typologies as discussed below exemplify how urban form and movement theories contribute to transport and land use planning policy and practice.

\subsubsection{Typologies for Transport and Land Use}

Combining angular choice for high and low radius identified which areas are well integrated into the local street network and enjoy good accessibility on the city scale. Nine typologies were identified based on the aggregate choice matrix (see Table 3). In Bergen, routes such as Fjøsangerveien with medium-to-high transport energy usage corresponds to routes with medium-to-high values for angular choice at a city-wide scale with medium-to-low angular choice values at local scale (types HM, ML, and HL). This is similar for Zürich (e.g., Utoquai) as well. The variable of angular choice shows through-movement potential or route-choice likeliness at the city-wide scale $(R=5000 \mathrm{~m})$. Regardless of to-movement potential or destination likeliness, these tended to emphasize common routes or arterial roads where large volume of traffic (usually private vehicles) travel through. These major, central, and regional roads are main flows for both person and goods transportation and the high energy usage due to the volume and speeds reflect this.

The inverse was also true. Areas in both cities with many streets with medium-to-low angular choice values at city-wide scale and medium to high values at local scale $(R=500 \mathrm{~m})$ enjoyed a lower energy usage pattern. These areas typically contain narrow streets and economic attractive areas for pedestrian, residential, and retail activities. The Sandviken area in Bergen and the Altstadt in Zürich are examples of these district roads, central and neighbourhood streets (types MH, LH, LM).

\subsubsection{Contributions to Policy and Practice}

This approach allows for a clarification of the relation between spatial configurations and transport energy usage. The use of space syntax allows for a value free evaluation of a city and to correlate it to simulated and real-time transport energy usage. By combining the results from the latest space syntax analyses techniques with energy use for transport, and reveal the results with the theory of the natural movement $[28,32]$ and the natural urban transformation process, the findings highlight what kinds of spatial configurations of the street network that are the necessary conditions to support and facilitate sustainable transport and land use patterns in existing cities. This assessment approach allows policymakers and practitioners to (i) identify potential locations in their cities to support such modes and what to avoid, and (ii) to provide insights into what type of spatial configurations would facilitate vibrant and lively urban areas.

Spatial configurations that facilitate and accommodate vehicular traffic tend towards higher transport energy usage while those that facilitate pedestrian activities or cycling or public transport tend towards lower transport energy usage. The former areas with low local scale angular choice values perpetuate private vehicle dependency and monofunctional areas of low density that become part of the urban sprawl. This creates complex and unsustainable transport patterns for travel for work, shopping, leisure activities and home. In addition, this might exacerbate transport inequalities 
for those who have access to private vehicles and those who do not [21,41]. The latter are areas with short urban blocks and tend to enjoy both high segment integration and angular choice values.

In line with Jane Jacobs [42], short urban blocks enhance walking or cycling as a transportation mode. Walking and cycling are modes with the lowest energy usage. Both modes are highly sought after in most cities in their pursuit of sustainable development. Both cases show that short urban blocks (or a fine-grained urban structure at local scale) with main routes running through them are necessary conditions for public transport and walkability. Areas with these conditions are highly sought after for enhancing sustainable transport outcomes. These areas also tend to evolve naturally into vibrant, highly urban areas with high building density and high degree of land use diversity supporting retail, commerce and social interaction [31,43]. Planners and policy makers therefore need to veer away from establishing new routes for heavy vehicular traffic only within the city core and enhance walkability (and slower modes) in current and potential socioeconomic centres. Concretely, this can be achieved by retrofitting current urban fabrics with the introduction of short urban blocks and fine meshed street networks $[11,20,43]$.

\section{Discussion and Reflection}

The above approach and findings contribute to novel insights to current academic discussions on transport and land use patterns in relation to energy consumption. The findings verify a positive correlation of car-oriented spatial configurations having higher energy usage. This is a critical element of addressing sustainability concerns, particularly in view of the energy transitions anticipated in the coming decades. In addition, the verification of what necessary conditions are required to support walkable, liveable and vital urban areas for those with and without access to private vehicles contributes to larger societal goals of creating and maintaining sustainable communities and cities. space syntax, which stems from precise concepts of urban space, applicable independent of cultural, economic, social or aesthetic contexts, allows for a value-free evaluation of the city. Moreover, it is possible to calculate spatial relationships independent from socioeconomic data with the space Syntax method. The MATSim model aggregates traffic data on the mobility network based on the place-bounded data regarding the location of urban functions. Therefore, overlapping and correlating these two models contributes to knowledge on the relation between urban spatial configuration and energy use for transport.

Planners and policy makers can use the findings to understand how spatial configurations affect transport energy usage. In addition, the approach using predominantly open sourced map data can be applied in various cities and regions across the world. Knowing the necessary conditions for walkable and liveable areas enables practitioners to plan and design for more integrated streets, differentiate which streets are desirable and which are less, and contribute additional savings in energy usage when the desired change is implemented. What the findings from Bergen and Zürich show, is that urban areas with short urban blocks or a fine-meshed street network with a well-connected main route running through these areas enhance low energy use for transport. The disclosure of high or low energy usage streets and areas based on a value-free evaluation of the city can also promote institutional innovation towards more sustainable mobility [44].

\subsection{Limitations}

The proposed approach experiences limitations in (i) the availability of transport energy data in different contexts for different modes, (ii) the simulation accuracy of energy data, (iii) complex operations in the joining of analysis across multiple platforms and (iv) replicability of results due to the previous limitations.

Data on energy registrations for walking and cycling are limited. Walking as a transportation mode is more complex than vehicular transport. A challenge for future research is to add registrations for pedestrian flow into the current approach and simulation models. Likewise, energy use per person for public transport such as trams and busses are more complex but need to eventually to be considered. 
As calculations will differ between private cars and transport of public goods or public transport or walking, the challenge is to build an aggregated model that does not become overly complex, if the data are available in the first place. In this article, Bergen data acquired were proprietary and only available to the authors as part of a research project. Whereas in Zürich, the data had to be simulated as it was otherwise not available.

Regarding the energy usage simulations, they are at best an approximation of actual consumption. As discussed in selecting variables for transport energy usage, energy losses for braking and changing speed and direction at stops, turns, inclines and more were left out of the equation. Vehicle production energy usage and other costs were also left out. In this simulation, only passenger transport was considered and not goods transport. The latter is a key cause of pollution and emissions in the EU and should be considered in future studies. From this perspective, it could be that real values could turn out to be (much) higher. On the other hand, current technological innovations have also led to more energy-efficient cars, which may result in lower values. The agent-based simulation's accuracy is therefore highly dependent on available input. This may explain slight differences in correlation significance between Bergen and Zürich, as measured traffic data was used in Bergen instead of a simulation. Further research could include dynamic, real-time traffic observations to resolve this limitation.

The novel contribution in correlating spatial configurations to transport energy usage required a complex, cross-platform analysis. The spatial configurations were analysed in DepthMap, the traffic values in MATSim and then both combined and geo-referenced in ArcMap. The analysis is therefore limited by potential join errors and data transference between platforms. Both Bergen and Zürich have one or more arterial roads that lead into or through the city via a tunnel. On some occasions, the terrain above is built up with streets and buildings, leading to errors in the spatial join operation where the energy values from the tunnel road were joined instead to the residential streets above. This had to be manually sieved out by the authors and the inaccuracy was (at least partly) corrected by taking out the $10 \%$ outliers in Zürich, see Table 4 .

The cross-comparison between cities allows for external validation of the findings. Although the approach operates from the value-free Space Syntax method, it is impossible to interpret the findings without local knowledge and field confirmation of the findings. Both case cities are similar in their historical development, urban structure and territorial conditions. To make the findings more robust, it is advisable to test the replicability of the approach in more cities and regions. This is of course subjected to data availability and access to the analytical tools used.

\subsection{Reflections}

The foremost use of the integrated assessment approach presented in this paper is a first step to build an energy classification for different street and road types. However, this model needs testing on other cities before making it operational for evaluating and diagnosing urban plans. At the very least, this model is a first step to understanding what the necessary conditions are for certain spatial configurations to achieve sustainable mobility for sustainable communities and cities.

It is not the intention of this article to villainize those who own and use private vehicles or to blame planning decisions for reinforcing unsustainable travel patterns and high energy consumption. In fact, one of the most vital transport flows i.e., goods and services have been unfortunately neglected due to lack of data availability. As long as the forces of a free market society prioritize profit-maximizing, mass-production, outsourcing and monoculture over energy efficiency, local economy, ecological diversity and social equity, a simple correlation of spatial configuration to energy usage remains limited in its ability to change the status quo. Here, the approach neglects who (a blue-collar worker or a CEO) is consuming transport energy.

In light of equity, health and well-being discussions in the SDGs, a potential future avenue of research would be to focus on who benefits and who pays in our current transportation system. With a shift towards thinking more in terms of local production and consumption in mind, further research 
should provide new insights how to achieve the Sustainable Development Goals within the transport and land use systems that we currently have.

Author Contributions: Conceptualization, all.; Data curation, all.; Formal analysis, R.d.K.; Funding acquisition, A.v.N.; Investigation, R.d.K., A.v.N.; Methodology, R.d.K., W.G.Z.T. and A.v.N.; Supervision, A.v.N.; Validation, W.G.Z.T. and R.d.K.; Writing - original draft, all. All authors have read and agreed to the published version of the manuscript.

Funding: This research is a part of the EU funded project SPACERGY-Space-Energy patterns for smart energy infrastructures, community reciprocities \& related governance (2016-2019)

Acknowledgments: Many thanks to Henrik Becker and Andreas Farner of the Institute for Transport Planning and Systems and the Center for Information Sciences and Databases of the ETH Zürich for the creation of the traffic simulation model and the cooperation in the Spacergy project. Likewise, many thanks to our student assistant Ine Askevold Hansen and Katrine Totland Hartvedt of Western Norway University of Sciences for mapping the microscale values for the Hochschulquartier and the Center of Bergen.

Conflicts of Interest: The authors declare no conflict of interest.

\section{References}

1. UN HABITAT. World Cities Report 2016; UN Habitat: Nairobi, Kenya, 2017; pp. 1-264.

2. European Environment Agency (EEA). Greenhouse Gas Emissions from Transport. Available online: https://www.eea.europa.eu/data-and-maps/indicators/transport-emissions-of-greenhouse-gases/ transport-emissions-of-greenhouse-gases-11 (accessed on 11 April 2019).

3. United Nations (UN). Resolution Adopted by the General Assembly on 6 July 2017. A/RES/71/313; Seventy-first Session, NY, USA. 2017. Available online: https://undocs.org/A/RES/71/313 (accessed on 11 April 2019).

4. Newman, P.W.; Kenworthy, J.R. Cities and Automobile Dependence: An International Sourcebook; Gower: Aldershot Hants, UK, 1989.

5. Nichols, B.G.; Kockelman, K.M. Urban form and life-cycle energy consumption: Case studies at the city scale. J. Transp. Land Use 2015, 8, 115-128. [CrossRef]

6. Papa, R.; Gargiulo, C.; Angiello, G. The Factors Influencing Transport Energy Consumption in Urban Areas: A Review. TeMA J. Land Use Mobil. Environ. 2014, 737-747. [CrossRef]

7. Steemers, K. Energy and the city: Density, buildings and transport. Energy Build. 2003, 35, 3-14. [CrossRef]

8. Hillier, B. Space is the Machine; Cambridge University Press: Cambridge, UK, 1996.

9. Hillier, B.; Hanson, J. The Social Logic of Space; Cambridge University Press: Cambridge, UK, 1984; pp. $108-109$.

10. Lefèvre, B. Urban Transport Energy Consumption: Determinants and Strategies for its Reduction. An analysis of the literature. Sapi En. S. Surv. Perspect. Integr. Environ. Soc. 2009, 2, 1-10.

11. Crane, R. The Influence of Urban Form on Travel: An Interpretive Review. J. Plan. Lit. 2016, 15, 3-23. [CrossRef]

12. Jabareen, Y.R. Sustainable Urban Forms. J. Plan. Educ. Res. 2016, 26, 38-52. [CrossRef]

13. Rådberg, J. Towards a theory of sustainability and urban quality; A new method for typological urban classification. In Proceedings of the 14th Conference of the International Association for People-environment Studies, Stockholm, Sweden, 30 July-3 August 1996; pp. 384-392.

14. Banister, D. The sustainable mobility paradigm. Transp. Policy 2008, 15, 73-80. [CrossRef]

15. Tan, W.G.Z. The Missing Link: Sustainable Mobility for Sustainable Cities and Communities. In Sustainable Development Goals in Southeast Asia and ASEAN; Holzhacker, R., Agussalim, D., Eds.; Brill: Leiden, The Netherlands, 2019; pp. 210-231. [CrossRef]

16. Wegener, M.; Fürst, F. Land-Use Transport Interaction: State of the Art; European Commission: Dortmund, Germany, 1999.

17. Derrible, S.; Kennedy, C. Characterizing metro networks: State, form, and structure. Transportation 2009, 37, 275-297. [CrossRef]

18. Siksna, A. The effects of block size and form in North American and Australian city centres. Urban Morphol. 1997, 1, 19-33.

19. Xie, F.; Levinson, D. Modeling the Growth of Transportation Networks: A Comprehensive Review. Netw. Spat. Econ. 2007, 9, 291-307. [CrossRef] 
20. Salingaros, N. Principles of Urban Structure; Techne Press: Delft, The Netherlands, 2005.

21. Hidayati, I.; Tan, W.; Yamu, C. How gender differences and perceptions of safety shape urban mobility in Southeast Asia. Transp. Res. Part F 2020, 73, 155-173. [CrossRef]

22. Moudon, A.V. Urban morphology as an emerging interdisciplinary field. Urban Morphol. 1997, 1, 3-10.

23. Hillier, B.; Penn, A.; Hanson, J.; Grajewski, T.; Xu, J. Natural Movement: Or, configuration and attraction in urban pedestrian movement. Environ. Plan. B 1993, 20, 29-66. [CrossRef]

24. Hillier, B.; Iida, S. Network and Psychological Effects in Urban Movement. In Proceedings of the Spatial Information Theory: International Conference, COSIT, Ellicottsville, NY, USA, 14-18 September 2005; pp. $475-490$.

25. van den Hoek, J. The mixed use index (MXI) as planning tool for (new) towns in the 21st century. In New Towns for the 21st Century: The Planned vs the Unplanned City; SUN: Amsterdam, The Netherlands, 2009; pp. 198-207.

26. van Nes, A. The Configurable Urban Sustainability. In what ways a morphological or configurational approach contributes to our understanding of urban sustainability? In Proceedings of the Open Building Conference, Hong Kong University, Hong Kong, China, 13-17 October 2003.

27. Van Nes, A.; Berghauser Pont, M.; Mashhoodi, B. Combination of space syntax with spacematrix and the mixed use index. In Proceedings of the 8th International Space Syntax Symposium, Santiago de Chile, Santiago, Chili, 3-6 January 2012.

28. Hillier, B.; Turner, A.; Yang, T.; Park, H.T. Metric and topo-geometric properties of urban street networks. In Proceedings of the 6th International Space Syntax Symposium, Istanbul, Turkey, 12-15 June 2007; Volume 1, pp. 1-22.

29. van Nes, A.; Yamu, C. Exploring Challenges in Space Syntax Theory Building: The Use of Positivist and Hermeneutic Explanatory Models. Sustainability 2020, 12, 7133. [CrossRef]

30. van Nes, A.; Stolk, E. Degrees of sustainable location of railway stations: Integrating Space syntax and Node place value model on railway stations in the province Noord-Holland's strategic plan for 2010-2040. In Proceedings of the 8th International Space Syntax Symposium, Santiago de Chile, Santiago, Chili, 3-6 January 2012.

31. Ye, Y.; van Nes, A. Quantitative tools in urban morphology: Combining space syntax, spacematrix and mixed-use index in a GIS framework. Urban Morphol. 2014, 18, 97-118.

32. Penn, A.; Hillier, B.; Banister, D.; Xu, J. Configurational modelling of urban movement networks. Environ. Plan. B 1998, 25, 59-84. [CrossRef]

33. Hjorthol, R.; Engebretsen, Ø.; Uteng, T.P. Den Nasjonale Reisevaneundersøkelsen 2013/14-Nøkkelrapport. TØI Rapport 1383/2014. 2014. Available online: https://www.toi.no/getfile.php/13395111441362561/Publikasjoner/T\T1\OI\%20rapporter/2014/1383-2014/1383-2014-elektronisk.pdf (accessed on 7 September 2020).

34. Stadt, Z. Stadtverkehr 2025, Strategie Für Eine Stadtverträgliche Mobilität 2014. Beilage 1 zu STRB Nr. 283/2014. Available online: https:/www.stadt-zuerich.ch/content/dam/stzh/ted/Deutsch/stadtverkehr2025/ Publikationen_und_Broschueren/Strategie_Stadtverkehr.pdf (accessed on 7 September 2020).

35. Karimi, K. Continuity and Change in Old Cities: An Analysis Investigation of the Spatial Structure in Iranian and English Historic Cities before and after Modernisation. Ph.D. Thesis, The Bartlett School of Graduate studies, University College London, London, UK, 1998.

36. de Koning, R.E.; van Nes, A.; Roald, H.-J.; Ye, Y. Strategies For Integrated Densification With Urban Qualities. Combining Space Syntax With Building Density, Land Use, Public Transport And Property Rights In Bergen City. In Proceedings of the 11th International Space Syntax Symposium, Lisbon, Portugal, 3-4 July 2017; pp. 863-879.

37. Rashid, M. The Geometry of Urban Layouts; Springer: Berlin/Heidelberg, Germany, 2017.

38. Horni, A.; Nagel, K.; Axhausen, K. The Multi-Agent Transport Simulation MATSim; Ubiquity Press: London, UK, 2016. [CrossRef]

39. MacKay, D. Sustainable Energy—Without the Hot Air; UIT: Cambridge, UK, 2009.

40. De Pascali, P.; Bagaini, A. Energy Transition and Urban Planning for Local Development. A Critical Review of the Evolution of Integrated Spatial and Energy Planning. Energies 2019, 12, 35. [CrossRef] 
41. Hidayati, I.; Yamu, C.; Tan, W. The Emergence of Mobility Inequality in Greater Jakarta, Indonesia: A Socio-Spatial Analysis of Path Dependencies in Transport-Land Use Policies. Sustainability 2019, 11, 5115. [CrossRef]

42. Jacobs, J. The Death and Life of Great American Cities; Pimlico: London, UK, 2000.

43. Gehl, J. Life between Buildings: Using Public Space; Island Press: Washington, DC, USA, 2011.

44. Homrighausen, J.R.; Tan, W.G.Z. Institutional Innovations for Sustainable Mobility: Comparing Groningen (NL) and Phoenix (US). Transp. Res. Procedia 2016, 19, 151-163. [CrossRef] 